\title{
The addition of an adductor canal block to local infiltration analgesia following total knee arthroplasty delays the attainment of physical therapy milestones
}

\author{
S. Dorman, G. Cuff, R. Cuevas, A. Atchabahian \\ NYU School of Medicine - New York (United States)
}

NYU School of Medicine

Background and Goal of Study: Prior studies and retrospective data have suggested an additive analgesic effect of adding an Adductor Canal Blockade (ACB) to Local Infiltration Analgesia (LIA) compared to LIA alone. We endeavored to determine whether Physical Therapy (PT) milestones were reached faster with ACB + LIA vs. LIA alone.

Materials and Methods: Following IRB approval, ASA IIII patients scheduled for unilateral primary TKA were randomized to receive either ACB or a sham ACB (saline) in addition to the routine LIA administered by the surgeon. Exclusion criteria included chronic pain and substance abuse. Anesthetic (spinal) and postoperative pain management were standardized. ACB was performed at the mid-thigh level with $30 \mathrm{~mL}$ of $2.5 \mathrm{mg} / \mathrm{mL}$ bupivacaine with 1:200K epinephrine. LIA was performed by the surgeon using bupivacaine, ketorolac, epinephrine and morphine, as well as liposomal bupivacaine.

Data collected included passive and active ROM knee flexion and extension, quadriceps strength, bed mobility skill, transfer skill, 2-minute walk test, and pain complaints via VAS before, during and after physical therapy sessions, as well as total distance ambulated on the day of surgery as well as the first and second postoperative days, ability to climb stairs, and readiness for discharge.
Results: Preliminary results of 51 patients out of 150 planned to be enrolled are reported. While pain scores, measured as an AUC for the initial 48 hours, was not different between groups (198 \pm 75 vs. $196 \pm 79$ ), total distances ambulated and 2-minute walk tests suggested that the group that received the ACB in addition to LIA achieved less on PT at each of the time points than the group that received LIA alone (total distances ambulated: DOS: $109 \pm 94$ vs. $132 \pm 85$ ft; POD1am: $75 \pm 106$ vs. $287 \pm 78 \mathrm{ft}$; POD1pm: $100 \pm 141$ vs. $255 \pm 50 \mathrm{ft}$; POD2am: $125 \pm 177$ vs. $215 \pm 50 \mathrm{ft}$; 2-minute walk test: DOS: $55 \pm 51$ vs. $74 \pm 58$ ft; POD1am: $20 \pm 14$ vs. $139 \pm$ 134 ft; POD1pm: $45 \pm 64$ vs. $140 \pm 99$ ft; POD2am: $75 \pm$ 107 vs. $128 \pm 83 \mathrm{ft})$.

Discussion: Contrary to our expectations and to prior publications, patients who received a block were able to walk less at every one of the time points than the patients who received LIA alone, while pain scores were not different. We will complete the study and investigate the possible causes for this discrepancy.

While these preliminary results remain to be confirmed, these data suggest that in combination with multimodal pharmacological anesthesia, LIA alone is preferable to $L I A+A C B$.

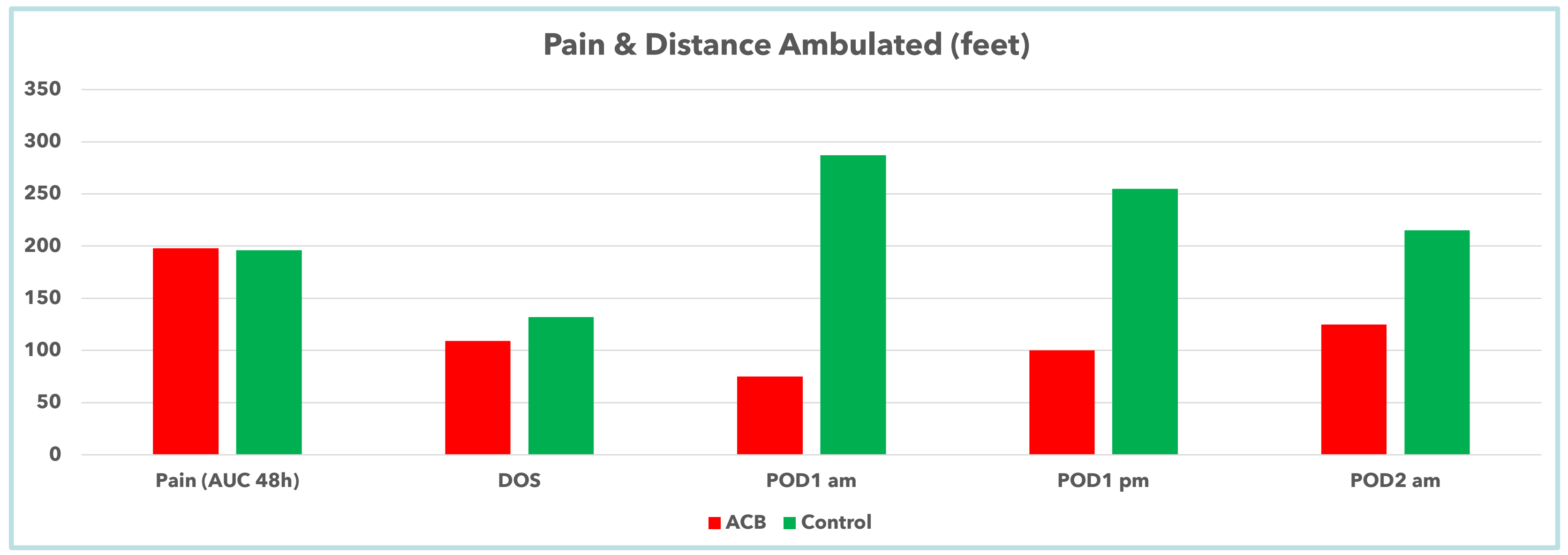

